\title{
A novel PIEZO1 mutation in a patient with dehydrated hereditary stomatocytosis: a case report and a brief review of literature
}

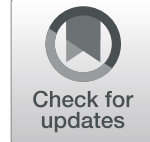

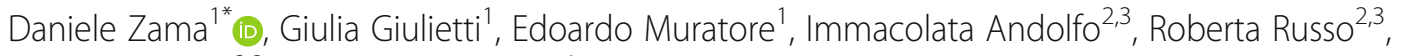
Achille Iolascon ${ }^{2,3}$ and Andrea Pession ${ }^{1}$

\begin{abstract}
Background: Dehydrated hereditary stomatocytosis (DHS) or hereditary xerocytosis is a rare, autosomal dominant hemolytic anemia characterized by macrocytosis, presence of stomatocytes and dehydration of red blood cells (RBCs). The dehydration is caused by a defect in cellular cation content. The most frequent expression of the pathology is hemolytic well-compensated anemia with high reticulocyte count, a tendency to macrocytosis, increased mean corpuscular hemoglobin concentration (MCHC) and mild jaundice. We here describe a new mutation of PIEZO1 gene, the most frequent mutated gene in DHS, in a family affected by hereditary hemolytic anemia.
\end{abstract}

Case presentation: We describe the case of a 12-years-old girl with well-compensated chronic hemolysis, increased MCHC and a father who had the same hematological characteristics. After excluding secondary causes of chronic hemolysis and enzymatic defects of the RBCs, microscopic observation of the peripheral blood smear, tests of RBC lysis, ektacytometry, SDS-PAGE and in last instance genetic analysis has been performed. This complex diagnostic workup identified a new variant in the PIEZO1 gene, never described in literature, causative of DHS. This pathogenetic variant was also detected in the father.

Conclusions: This case report highlights the importance of a correct and exhaustive diagnostic-workup in patients with clinical suspicious for hemolytic anemia in order to make a differential diagnosis. This is relevant for the management of these patients because splenectomy is contraindicated in DHS due to high thrombotic risk.

Keywords: Hemolytic anemia, Dehydrated hereditary stomatocytosis, Mean corpuscular hemoglobin concentration (MCHC), Genetic disease, Next generation sequencing (NGS)

\section{Background}

Dehydrated hereditary stomatocytosis (DHS) or hereditary xerocytosis (OMIM\#194380) is a rare, autosomal dominant congenital hemolytic anemia, characterized by large and dehydrated red blood cells (RBCs) [1]. DHS is the most common type among the large group of

\footnotetext{
* Correspondence: daniele.zama@gmail.com

'Department of Pediatrics, "Lalla Seràgnoli," Hematology-Oncology Unit,

Sant'Orsola-Malpighi Hospital, University of Bologna, Via Massarenti 11, 40137 Bologna, Italy

Full list of author information is available at the end of the article
}

hereditary stomatocytosis. It is $10-20$ times less frequent than hereditary spherocytosis, and shares with it some common features, leading to possible missdiagnosis [1].

It is caused by an alteration of the RBC membrane permeability to the monovalent cations $\mathrm{Na}+$ and $\mathrm{K}+$, with a consequent alteration of the intracellular cationic content, cell dehydration, and modifications of cell volume. The main causative gene is PIEZO1 [2], localized at 16q23-24. PIEZO1 (OMIM\#194380) encodes for a mechanoreceptor, that is a cation channel activated by 
various types of mechanical stimuli, and that functions as a biological pressure sensor in both vertebrates and invertebrates. It is high expressed at plasma membrane of RBCs in which it regulates the cell volume [3-6]. The identified mutations are mostly missense and mainly located in the highly conserved C-terminus of the protein, recently described to form the pore of the channel [7]. Several electrophysiology studies demonstrated that the mutations cause a gain-of-function phenotype with delayed inactivation of the channel $[2,8,9]$, explaining the increased cation permeability that leads to erythrocyte dehydration. Mutations of KCNN4 (OMIM\#616689), encoding the Gardos channel, a widely expressed $\mathrm{Ca} 2+$ -dependent $\mathrm{K}+$ channel of intermediate conductance that mediates the major $\mathrm{K}+$ conductance of erythrocytes have also been described recently in literature as causative of DHS2 [10, 11].

The clinical phenotype of DHS ranges from asymptomatic to severe forms, with massive hemolysis. Sign and symptoms comprise mild to severe anemia, jaundice, pallor, fatigue, splenomegaly, gallstones, and severe iron overload $[1,7,9]$.

DHS patients generally show hemolytic wellcompensated anemia, with a high reticulocyte count, a tendency to macrocytosis and mild jaundice. The main characteristic of RBCs is cell dehydration caused by the loss of the cation content, with a subsequent increase of MCHC (>36 g/dL). At blood smear, the stomatocytes, erythrocytes with a characteristic central mouth-shaped spot [2], are quite rare, which often makes diagnosis difficult. Osmotic gradient ektacytometry is a useful and often critical examination to diagnose this condition. It shows a leftward shift of the osmolarity curve due to the presence of dehydrated erythrocytes [12].

DHS can be classified into a non-syndromic form, with only hematological involvement, and syndromic form, also called pleiotropic syndrome, characterized by anemia with pseudohyperkalemia and/or pre/peri-natal edema $[2,13,14]$. DHS is often undiagnosed or misdiagnosed with other conditions, especially hereditary spherocytosis.

\section{Case presentation}

The proband was a 12-years-old girl presenting with chronic compensated hemolysis, Gilbert syndrome, and recurrent abdominal pain. The blood count showed: hemoglobin $13 \mathrm{~g} / \mathrm{dL}$, red blood cell counts 4.020.000/ $\mathrm{mm}^{3}$, Mean Corpuscular Volume (MCV) $90.3 \mathrm{fL}$, Mean Content Hemoglobin $(\mathrm{MCH}) 32.1 \mathrm{pg}$, mean corpuscular hemoglobin concentration (MCHC) $38.3 \mathrm{~g} / \mathrm{dL}$, reticulocytosis, and indirect hyperbilirubinemia. The complete blood work-up is shown in Table 1. The ultrasound of the abdomen did not show gallstones, hepatomegaly and splenomegaly.
The patient was a single-born from nonconsanguineous Italian parents (Fig. 1a). The remote case history reported neonatal jaundice treated with phototherapy. The family anamnesis revealed that the father presented jaundice during his childhood, recurrent episodes of abdominal pain resolved after splenectomy (performed at the age of 23 years-old) and gallstones for which he had undergone a cholecystectomy. The paternal uncle also had gallbladder stones. To investigate the cause of hemolysis in our patient, laboratory investigations were carried out. The peripheral blood smear showed anisocytosis with the presence of red blood cells with specific shape: stomatocytes (5\%), ovalocytes (4\%), schistocytes (3\%) and rare spherocytes (Fig. 1b). The direct antiglobulin test (DAT) was negative, thus excluding an autoimmune origin of the hemolysis. Structural hemoglobin alterations have also been excluded performing electrophoresis and molecular assessment of the genes encoding beta and alpha-globin chains. The activity of red blood cell metabolism enzymes (Hexokinase, Glucose-6-phosphate isomerase, 6-Phosphofruttochinase, Glyceraldehyde phosphate dehydrogenase, Phosphoglycerate kinase, Pyruvate kinase, Glucose 6 phosphate dehydrogenase, Adenylate 6Phosphogluconate dehydrogenase kinase) was normal.

The family history showed an autosomal dominant inheritance of the condition. To investigate the possible presence of a spherocytosis condition, the most frequent erythrocyte structural defect, a combination of tests was performed. First, the eosin- $5^{\prime}$-maleimide binding test (EMA test), a cytometric analysis in which a fluoresceinated (eosin-5' -maleimide) substance binds to the plasma membrane proteins of red blood cells, mainly to the band 3 protein [1,9]. The average fluorescence of RBCs with EMA staining in patients with spherocytosis is lower than that of control RBCs due to the decrease in the number of target proteins. In our case, this examination was in the normal range with a value of $12 \%$ (normal test value> $11 \%)$. The other complementary investigations carried out were the osmotic resistance tests such as the glycerol lysis test (AGLT50) and the Pink test. These tests were normal in our patient. Furthermore, quantitative analysis of membrane proteins was carried out using Sodium Dodecyl Sulphate-PolyAcrylamide Gel Electrophoresis (SDSPAGE), and resulted not altered. We also performed the ektacytometry that evaluates the erythrocyte deformability by subjecting them to an increasing osmotic gradient with constant shear stress [12]. Ektacytometry showed a left shift of the osmolarity curve suggestive of DHS. In agreement with the clinical suspicion, genetic testing was carried out both in the patient and in the parents by a targeted-NGS custom panel composed of 86 causative genes of hereditary anemias. This panel is an updated version of a similar previously published one [15]. We found 
Table 1 Complete blood work-up of the patient

\begin{tabular}{|c|c|c|}
\hline & Value & Reference Range \\
\hline Red blood cell count ( $\mathrm{n} / \mathrm{mmc}$ ) & 4.020 .000 & $4.200 .000-6.100 .000$ \\
\hline Hemoglobin (g/dl) & 13 & $12-18$ \\
\hline Hematocrit (\%) & 36,3 & $37-52$ \\
\hline Mean Corpuscolar Volume (fL) & 90,3 & $80-99$ \\
\hline Mean Corpuscolar Hemoglobin (pg) & 32,1 & $27-31$ \\
\hline Mean Corpuscolar Hemoglobin Concentration (g/dL) & 38,3 & $33-37$ \\
\hline Red-Cell distribution Widht (\%) & 12,9 & $11,5-14,5$ \\
\hline Reticulocyte count (/mmc) & 178.000 & $22.000-139.000$ \\
\hline Reticulocyte count \% (\%) & 4,43 & $0,5-2,5$ \\
\hline Mean Corpuscolar Hemoglobin Concentration of Reticulocyte (g/dl) & 32,9 & $23-29$ \\
\hline Mean Corpuscolar Hemoglobin of Reticulocyte (pg) & 33,7 & $25-30$ \\
\hline Erythropoietin (mU/ml) & 33,8 & $2,6-18,5$ \\
\hline Total Bilirubin (mg/dl) & 8,58 & $<1,20$ \\
\hline Direct Bilirubin (mg/dl) & 0,72 & $<0,30$ \\
\hline Indirect Bilirubin (mg/dl) & 7,86 & $<0,90$ \\
\hline Haptoglobin (mg/dl) & $<30$ & $30-200$ \\
\hline Lactate Dehydrogenase (U/I) & 138 & 110-295 \\
\hline Hemoglobin A2 (\%) & 3,1 & $1,8-3,2$ \\
\hline Hemoglobin F (\%) & 4 & $<1$ \\
\hline Serum iron level (mcg/dl) & 193 & $60-18$ \\
\hline Transferrin (mg/dl) & 259 & $200-360$ \\
\hline Ferritin (ng/dl) & 43 & $11-306$ \\
\hline
\end{tabular}

in both subjects the missense variant c.1815G > A, p.Met605Ile in PIEZO1 gene (NM_001142864, CCDS54058) in heterozygous state. According to the guidelines of American College of Medical Genetics and Genomics (ACMG), we evaluated the pathogenicity of this variant by gathering evidence from various sources: population data, computational and predictive data, functional data, and segregation data [16]. First, the variant segregated in the affected father (Fig. 1a). Currently, this variant is annotated neither in population databases nor in databases of known variant. Moreover, it is predicted by several tools as probably damaging (MutationTaster score 0.999 Disease causing; FATH MM Score-1.59; LRT prediction Deleterious; PolyPhen2 score HumVar 0.968 Probably damaging; PROVEAN Score-2.95 Damaging).

\section{A}

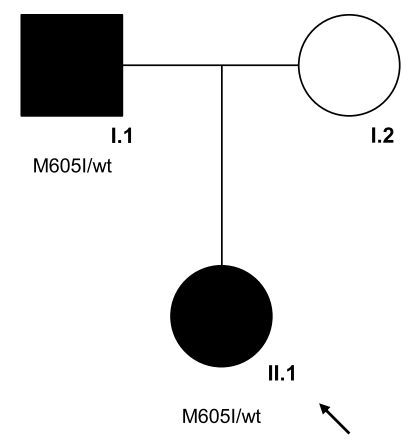

B

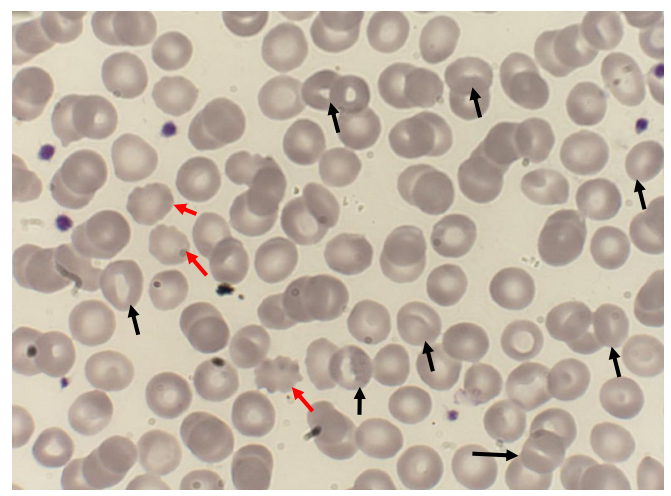

Fig. 1 a The family tree comprising the proband (black arrow), the affected father and the unaffected mother. b Morphological examination of peripheral blood showing typical stomatocytes (black arrows) and acanthocytes (red arrows) 


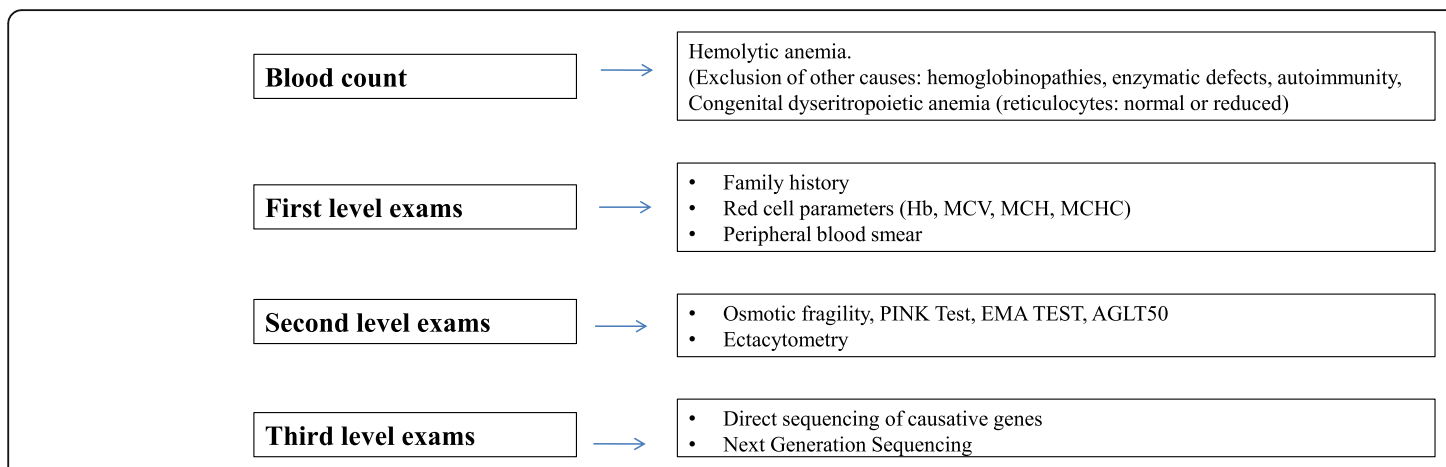

Fig. 2 Laboratory tests that guide the differential diagnosis [7]

\section{Discussion and conclusions}

Our case demonstrates the complexity of differential diagnosis of hemolytic anemias in pediatric patients. A correct diagnostic workup should consider autoimmunity, enzymatic defects of the RBCs and erythrocyte membrane alterations, including, spherocytosis, elliptocytosis, ovalocytosis of South Asia, pyropoikilocytosis and stomatocytosis. Secondary causes, such as liver disease, must be also ruled oute [17] (Fig. 2).

The first line investigation includes erythrocyte parameters (in particular $\mathrm{Hb}, \mathrm{MCV}, \mathrm{MCH}$, and $\mathrm{MHCH}$ ), observation of the peripheral blood smear, and evaluation of the family history. The second line includes EMA test, osmotic fragility test, AGLT-50 test, and the pink test, that can address the diagnosis. It is also crucial to carry out ektacytometry analysis which shows characteristics deformation of the red blood cells, allowing to guide the diagnosis towards a precise pathology of the erythrocyte membrane. The third diagnostic line is represented by the sequencing of the candidate causative genes or the contemporary sequencing of a panel of genes by Next Generation Sequencing (NGS) technique, that has allowed the identification of numerous causative mutations associated with defined morphological pictures, with reduced times and costs $[15,18,19]$.
Herein, we identified a novel PIEZO1 variant that is located in non-pore domain of the PIEZO1 mechanoreceptor, responsible for the mechanosensitive properties of the channel [7]. Our recent genotype-phenotype correlation analysis on 29 PIEZO1-patients demonstrated that most of severely affected patients carried mutations in the pore domain, responsible of the ion passage, while patients showing a less severe phenotype carried mutations in the nonpore domain [7]. In accordance to this finding, the patients here described, both proband and father, showed a mild phenotype and the absence of iron overload [20].

Regarding treatment, for stomatocytosis, as for other RBC membrane pathologies, therapy is phototherapy/ exanguino-transfusion in newborns suffering from this condition. Transfusions may also be necessary at any time of life due to aplastic and hemolytic crises.

It is particularly important to diagnose this pathology and to distinguish it from spherocytosis, because of the clinical consequences (Table 2). Indeed, patients with DHS have a higher risk of thromboembolic complications (portal thrombosis and pulmonary hypertension) [21] after splenectomy than in spherocytosis, due to an increased number of stomatocytes, not destroyed in the

Table 2 DHS and spherocytosis in comparison

\begin{tabular}{lll}
\hline & DHS & Spherocytosis \\
\hline MCHC & Increased & Increased \\
RDW & Increased & Increased \\
MCV & Tendency to macrocytosis & Normal or slightly reduced \\
$\begin{array}{l}\text { Peripheral blood } \\
\text { smear }\end{array}$ & Stomatocytes & Spherocytes \\
$\begin{array}{l}\text { Splenomegaly } \\
\text { EMA test and RBCs } \\
\text { lysis tests }\end{array}$ & mild / moderate & Normal/Increased \\
Ectacytometry & Normal Elmax and left shift of Omin and OHyper due to the & Variable from mild to severe \\
& presence of dehydrated red cells. & Decreased \\
Causative genes & PIEZO1/ KCNN4 & (reduced surface / volume ratio) and shift to the left \\
\hline
\end{tabular}


spleen, present in systemic circulation. Splenectomy is thus contraindicated in this condition. In addition, in patients with DHS iron metabolism should be regularly checked for the risk of developing sever hepatic iron overload [20] (Table 2).

In conclusion, our case report shows that the diagnosis of DHS can be difficult and there can be a diagnostic delay if it is not properly suspected. Thus, we advise the use of a structured and exhaustive diagnostic workup. The application of a precise workflow led us to discover a new causative variant not yet described, highlighting the importance of a complete genetic workup in this category of patients.

\begin{abstract}
Abbreviations
ACMG: American College of Medical Genetics and Genomics; AGLT50: Acidified glycerol lysis test; DHS: Hereditary dehydrated stomatocytosis; EMA test: Eosin-5'-maleimide binding test; MCH: Mean Content Hemoglobin; MCHC: Mean corpuscular hemoglobin concentration; MCV): Mean corpuscular volume; RBC: Red blood cell; RDW: Red blood cells distribution width; SDS-PAGE: Sodium Dodecyl Sulphate - PolyAcrylamide Gel Electrophoresis
\end{abstract}

\section{Authors' contributions}

All the Authors have revised the manuscript and contributed to the drafting of the article. They confirm that the manuscript is an original version and has not been published in any other scientific Journal or elsewhere. All authors read and approved the final manuscript.

\section{Funding}

This work was supported by EHA Junior Research Grant to Immacolata Andolfo (3978026), and by Bando Star Linea 1 - JUNIOR PRINCIPAL INVESTIG ATOR GRANTS - COINOR, Università degli Studi di Napoli Federico II to Roberta Russo.

\section{Availability of data and materials}

Data sharing not applicable to this article as no datasets were generated or analysed during the current study. The data used and/or analyzed during the writing of this manuscript are available from the corresponding Author on reasonable request.

\section{Ethics approval and consent to participate}

No ethical committee approval is required for this case report. Authors obtained written informed consent for publication from the patient's parents.

\section{Consent for publication}

Authors agree to submit the manuscript to the IJP for publication.

\section{Competing interests}

The authors declare that they have no competing interests.

\section{Author details}

'Department of Pediatrics, "Lalla Seràgnoli," Hematology-Oncology Unit, Sant'Orsola-Malpighi Hospital, University of Bologna, Via Massarenti 11, 40137 Bologna, Italy. ${ }^{2}$ Department of Molecular Medicine and Medical Biotechnologies, "Federico II" University of Naples, Naples, Italy. ${ }^{3}$ CEINGE, Biotecnologie Avanzate, Naples, Italy.

Received: 20 November 2019 Accepted: 10 July 2020

Published online: 23 July 2020

\section{References}

1. Andolfo I, Russo R, Gambale A, lolascon A. New insights on hereditary erythrocyte membrane defects. Haematologica. 2016;101:1284-94.

2. Andolfo I, Alper SL, De Franceschi L, Auriemma C, Russo R, De Falco L, et al. Multiple clinical forms of dehydrated hereditary stomatocytosis arise from mutations in PIEZO1. Blood. 2013;121:3925-35.
3. Ge J, Li W, Zhao Q, Li N, Chen M, Zhi P, et al. Architecture of the mammalian mechanosensitive Piezo1 channel. Nature. 2015;527:64-9.

4. Cinar E, Zhou S, DeCourcey J, Wang Y, Waugh RE, Wan J. Piezo1 regulates mechanotransductive release of ATP from human RBCs. Proc Natl Acad Sci. 2015;112:11783-8

5. Coste B, Xiao B, Santos JS, Syeda R, Grandl J, Spencer KS, et al. Piezo proteins are pore-forming subunits of mechanically activated channels. Nature. 2012;483:176-81.

6. Coste B, Mathur J, Schmidt M, Earley TJ, Ranade S, Petrus MJ, et al. Piezo 1 and Piezo2 are essential components of distinct mechanically activated cation channels. Science (80- ). 2010;330:55-60.

7. Andolfo I, Russo R, Rosato BE, Manna F, Gambale A, Brugnara C, Iolascon A. Genotype-phenotype correlation and risk stratification in a cohort of 123 hereditary stomatocytosis patients. Am J Hematol. 2018;93(12):1509-17.

8. Bae C, Gnanasambandam R, Nicolai C, Sachs F, Gottlieb PA. Xerocytosis is caused by mutations that alter the kinetics of the mechanosensitive channel PIEZO1. Proc Natl Acad Sci. 2013;110:E1162-8.

9. Iolascon A, Andolfo I, Russo R. Advances in understanding the pathogenesis of red cell membrane disorders. Br J Haematol. 2019;187:13-24.

10. Andolfo I, Russo R, Manna F, Shmukler BE, Gambale A, Vitiello G, De Rosa G, Brugnara C, Alper SL, Snyder LM, Iolascon A. Novel Gardos channel mutations linked to dehydrated hereditary stomatocytosis (xerocytosis). Am J Hematol. 2015;90(10):921-6.

11. Rapetti-Mauss R, Lacoste C, Picard V, Guitton C, Lombard E, Loosveld M, Nivaggioni V, Dasilva N, Salgado D, Desvignes JP, Béroud C, Viout P, Bernard M, Soriani O, Vinti H, Lacroze V, Feneant-Thibault M, Thuret I, Guizouarn H, Badens C. A mutation in the Gardos channel is associated with hereditary xerocytosis. Blood. 2015;126(11):1273-8012.

12. Llaudet-Planas E, Vives-Corrons JL, Rizzuto V, Gómez-Ramírez P, Sevilla Navarro J, Coll Sibina MT, García-Bernal M, Ruiz Llobet A, Badell I, VelascoPuyó P, Dapena JL, Mañú-Pereira MM. Osmotic gradient ektacytometry: A valuable screening test for hereditary spherocytosis and other red blood cell membrane disorders. Int J Lab Hematol. 2018;40(1):94-102.

13. Grootenboer-Mignot S, Crétien A, Laurendeau I, Poissonnier M-H, Doireau V, Brossard $Y$, et al. Sub-lethal hydrops as a manifestation of dehydrated hereditary stomatocytosis in two consecutive pregnancies. Prenat Diagn. 2003:23:380-4.

14. Grootenboer S, Schischmanoff PO, Laurendeau I, Cynober T, Tchernia G, Dommergues JP, et al. Pleiotropic syndrome of dehydrated hereditary stomatocytosis, pseudohyperkalemia, and perinatal edema maps to 16q23q24. Blood. 2000;96:2599-605.

15. Russo R, Andolfo I, Manna F, Gambale A, Marra R, Rosato BE, et al. Multigene panel testing improves diagnosis and management of patients with hereditary anemias. Am J Hematol. 2018;93:672-82.

16. Richards S, Aziz N, Bale S, Laboratory Quality Assurance Committee ACMG, et al. Standards and guidelines for the interpretation of sequence variants: a joint consensus recommendation of the American College of Medical Genetics and Genomics and the Association for Molecular Pathology. Genet Med. 2015;17:405-24.

17. Da Costa L, Galimand J, Fenneteau O, Mohandas N. Hereditary spherocytosis, elliptocytosis, and other red cell membrane disorders. Blood Rev. 2013;27:167-78.

18. Kim Y, Park J, Kim M. Diagnostic approaches for inherited hemolytic anemia in the genetic era. Blood Res. 2017;52:84-94.

19. Agarwal AM, Nussenzveig RH, Reading NS, et al. Clinical utility of nextgeneration sequencing in the diagnosis of hereditary haemolytic anaemias. Br J Haematol. 2016;174(5):806-14 https://doi.org/10.1111/bjh.14131.

20. Andolfo I, Rosato BE, Manna F, De Rosa G, Marra R, Gambale A, et al. Gainof-function mutations in PIEZO1 directly impair hepatic iron metabolism via the inhibition of the BMP/SMADs pathway. Am J Hematol. 2020;95:188-97.

21. Jaïs X, Till SJ, Cynober T, loos V, Garcia G, Tchernia G, et al. An extreme consequence of splenectomy in dehydrated hereditary stomatocytosis: gradual thrombo-embolic pulmonary hypertension and lung-heart transplantation. Hemoglobin. 2003;27:139-47.

\section{Publisher's Note}

Springer Nature remains neutral with regard to jurisdictional claims in published maps and institutional affiliations. 